\title{
«L'érotisme des autres » : phénoménologie de la pornographie chez Robbe-Grillet
}

\author{
Andrea Chiurato \\ Université IULM de Milan
}

La pornographie peut corrompre une société et une civilisation. Les élus politiques ont le droit et l'obligation d'éviter cette corruption.

Richard Nixon, Déclaration sur le rapport de la Commission sur les obscénités et la pornographie

Sur quoi veillent nos censeurs? Sur la pureté morale des nations. Jusque-là, tout va bien, après tout, chacun son travail. Mais ils prétendent aussi, et c'est alors que les choses se compliquent, lutter contre l'extension du crime. Alain Robbe-Grillet, Angélique ou l'enchantement 
Dans son essai I limiti dell'estetica, Maddalena Mazzocut-Mis affirme que les limites de l'art sont définies par trois frontières expérientielles: l'horreur, le dégoût et l'obscénité. En fait, affronter ce qui est impur est un élément important pour la compréhension de l'esthétique moderne. Ce point de départ permet de mettre en évidence la réaction contradictoire (embarras et fascination à la fois) que l'impur déclenche chez le spectateur, lequel crée « une véritable fracture entre lui-même et l'objet représenté : il désapprouve l'objet de la représentation, mais il tire une intime satisfaction de sa jouissance » (Mazzocut-Mis, p. 199) ${ }^{1}$. Ce paradoxe est déterminé par plusieurs facteurs, qui doivent être pris en compte de manière très attentive.

On doit, d'abord, analyser l'effet produit sur le public par la représentation ainsi que la relation entre l'artiste et les institutions qui, historiquement, ont lutté contre l'obscénité. Parallèlement à cette perspective sociologique, il existe la question esthétique : dans le premier cas, on peut se demander ce qui est mis en scène, mais nous devons également comprendre comment cela est représenté. Le troisième point implique une autre perspective: se pencher sur la relation entre impureté et inconscient signifie aborder le domaine de la psychologie. Aujourd'hui, à l'époque post-freudienne qui est la nôtre, nous ne pouvons pas ignorer la relation entre les thèmes scandaleux et les tabous sociaux. Dans cette perspective,

\footnotetext{
${ }^{1}$ « La realtà diventa sua rappresentazione ed egli [lo spettatore, n.d.a.] crea una vera e propria frattura tra se stesso e l'oggetto rappresentato: l'oggetto riceve la disapprovazione, ma la sua fruizione provoca piacere.». Nous traduisons.
} 
l'impur est simplement une étiquette qui sert à définir ce qui existe au-delà des limites de la conscience et de la normalité.

Isoler ces trois perspectives est absolument nécessaire et utile sur le plan pratique, notamment si nous pensons aux sérieuses conséquences qu'une confusion parmi ces différents points de vue pourrait engendrer: scandales, procès et condamnations sévères. Le fantôme du marquis de Sade hante toujours nos esprits, mais il n'est pas nécessaire d'aller jusquelà pour trouver des cas semblables.

\section{L'artiste sur la scène du crime}

En 1975, Alain Robbe-Grillet a été condamné par le tribunal civil de Venise pour son film Glissements progressifs du plaisir. Le parquet a fondé son accusation sur un argument très simple : l'exhibition de scènes indécentes dans un film ne peut être tolérée que si elle est justifiée par les «nécessités de l'intrigue ». Quand l'artiste ignore ces nécessités, il n'est plus en train d'exercer son droit à la liberté d'expression, mais de camoufler la pornographie sous l'apparence de l'érotisme. L'attitude du parquet dissimule un point de vue plutôt naïf : tout comme les oiseaux de l'Historia naturalis de Pline l'Ancien, il a tendance à confondre réalité et illusion. Dans ce mythe, en effet, les oiseaux, trompés par les belles peintures réalisées par Zeuxis, essaient de manger les raisins dessinés sur le mur. Dans les deux cas, il y a un manque de détachement entre le spectateur et ce qui est observé, et cette proximité est source de confusion. Comme le montre Nathalie Sarraute dans L'Ère du soupçon, sans le degré de détachement approprié, il n'y a pas de perception ni de plaisir esthétiques. 
Notre but n'est pas de mettre en question le jugement du tribunal. Nous rappelons seulement cet épisode parce qu'il s'agit d'un bon exemple de malentendu. Si l'on analyse certains mots utilisés par le tribunal, on a l'impression que les choses sont plutôt claires: les chefs d'accusation sont indiqués de manière très simple. Tout l'argument est axé sur des stéréotypes et des banalités: qui peut nier que la représentation du corps a toujours été considérée scandaleuse dans la civilisation occidentale?

Cependant, nous devons prendre en compte les modalités de la représentation, parce que la distinction entre érotisme et pornographie n'est pas déterminée par ce qui est représenté, mais plutôt par la manière dont cela est représenté. Le tribunal n'a pourtant pas suivi cet argument jusqu'à sa conclusion logique, notamment quand il a affirmé que toutes les scènes de nu devraient être justifiées par les «nécessités de l'intrigue ». Cela constitue un point fondamental, qui ouvre la porte à une vaste gamme d'interprétations. Tout à coup, on se trouve égaré dans une sorte de no man's land où l'art doit pactiser avec des institutions sociales qui lui sont étrangères. Le sens, généralement accepté, de certains termes ambigus, tels intrigue ou nécessité, est le résultat de cette négociation.

Dès le début de sa carrière, Robbe-Grillet a affiché une remarquable hostilité envers certaines "notions périmées", notamment envers l'un des principaux dogmes de la théorie narrative traditionnelle : l'histoire ou intrigue. Dans ses romans comme dans ses films, la séquence des scènes ne suit pas des lois logiques, chronologiques ou psychologiques, mais elle est souvent déterminée par un conflit intérieur. Cela est un élément intéressant, mais le parquet donne l'impression d'ignorer cet 
aspect technique, et l'arrêt du tribunal se fonde uniquement sur une conception de l'intrigue qui ne correspond pas à l'esthétique adoptée par le Nouveau Roman.

Nous voulons insister, une fois de plus, sur cette hypothèse implicite de l'argument du parquet: en l'absence d'une intrigue réelle, toutes les scènes de nu doivent être considérées, sans distinction, "obscènes». Selon la critique américaine Elisabeth Newton, le tribunal de Venise n'aurait pas pu choisir une meilleure cible :

Le film et la fiction d'Alain Robbe-Grillet sont remarquables pour le nombre de scènes et de descriptions incluant des représentations de la sexualité. La sexualité a toujours imprégné, dans une certaine mesure, les œuvres de RobbeGrillet; dès ses tous premiers romans [...] la tension sexuelle a toujours été présente, même sans être explicitement mentionnée. (p. 121) ${ }^{2}$

Homicide, torture, sadisme, pédophilie, cannibalisme, etc. : tout au long de sa carrière, Robbe-Grillet a exploré une pléthore de perversions. Dès ses premières œuvres, ses représentations sexuelles pour le moins audacieuses ont choqué les critiques. Après avoir lu Le Voyeur, en 1955, Émile Henriot a proposé d'enfermer le romancier à l'hôpital psychiatrique Sainte Anne. Les stratégies rhétoriques utilisées par le parquet et par le critique du Monde méritent d'être analysées de manière attentive.

\footnotetext{
2 «Alain Robbe-Grillet's film and fiction are remarkable for the amount of scenes or descriptions they contain that include depictions of sexuality. Sexuality has always suffused Robbe-Grillet's work to some extent; from his earliest novels [...] sexual tension has always been present, and yet is not explicitly referred to. ». Nous traduisons.
} 
Pour le tribunal, chaque «scène de nu » doit être justifiée par les «nécessités de l'intrigue». Au contraire, pour RobbeGrillet, ces mêmes scènes sont justifiées par un détachement significatif (une "forte distanciation») entre le public et la représentation. Dans le premier cas, la condamnation esthétique se fonde sur une inquiétude non littéraire (les effets sociaux de l'art), c'est-à-dire sur la conviction que la pornographie peut contribuer à la corruption morale. RobbeGrillet réfute cette accusation en prenant d'abord en considération des raisons esthétiques, et ce n'est que dans un deuxième temps qu'il exprime un jugement moral. Douze ans après, dans Angélique ou l'enchantement, il revient sur la scène du crime. Le premier témoin appelé pour sa défense est Sophocle :

Personnellement, je suis de ceux qui croient - comme Sophocle - à la valeur cathartique des représentations effectuées au grand jour. Mais, pour que cette fonction puisse opérer pleinement, les acteurs athéniens portaient des masques stéréotypés sur le visage, afin de détruire toute possibilité d'illusion réaliste. Il semble bien, en effet, qu'une forte distanciation ait de longue date été reconnue nécessaire (deux ou trois mille ans avant Brecht) pour éviter que le spectateur naï ou le lecteur, ne cède à la douceur de s'identifier sans réfléchir à l'acte représenté. Ce qu'il faut, c'est qu'il se regarde lui-même; une distance doit donc être marquée entre son corps et ce qu'on lui donne à voir, pour qu'une distance intérieure se fasse jour dans son propre esprit. (p. 198)

Selon Robbe-Grillet, tous les thèmes générateurs qui reviennent obsessionnellement dans ses œuvres («l'inceste, le parricide, le sacrifice des vierges, l'assassinat et la dégustation des enfants » (1987, p. 197) sont aussi les thèmes principaux de la tragédie grecque. En recourant à d'autres exemples de ce genre, il arrive à montrer, paradoxalement, que les représentants de la loi sont 
aveuglés par une sorte de myopie : ils font l'éloge des anciens Grecs pour leur "sagesse civique » et, en même temps, ils condamnent pour obscénité un artiste contemporain qui partage les mêmes contenus. La cause de cette différence d'attitude réside dans le fait que ses juges sont simplement mal placés : ils n’ont pas le degré de détachement convenable. Ils oublient que même le pire des films pornographiques raconte une histoire, mais ils ont l'air de préférer cette façon classique de raconter (en décrivant une action et son développement) plutôt que la nouvelle approche proposée, par exemple, par la Nouvelle Vague ${ }^{3}$. Dans le cinéma expérimental des années 1960, il n'y a pas en effet de véritable développement, mais seulement une série de possibilités alternatives. On n'arrive pas à saisir le sens de l'histoire parce que ce sens demeure toujours ambigu.

Pour continuer avec les paradoxes, l'argument de RobbeGrillet transforme l'accusation d'anti-humanisme en une arme puissante. Si les personnages traditionnels ne trouvent pas de place dans ses films et ses romans (réduits à une coquille vide, privés de toute profondeur psychologique, etc.), comment le public pourrait-il partager leur souffrance? De fait, l'attention maniaque que le Nouveau Romancier, dans ses descriptions, porte aux détails ne suscite pas de réponse émotionnelle, mais elle est, au contraire, un instrument très utile pour détruire toutes les illusions de la réalité : «les représentations de provocations féminines (suivies de punitions ou des martyres) sont [en fait] figées et dénaturalisées par le processus d'écriture qui mettent en scène l'illusion du réalisme : leur caractère de

\footnotetext{
${ }^{3}$ Selon Eco (p. 130-131), on peut trouver beaucoup d'« action » dans ces films, mais il n'y a pas de véritable « intrigue ».
} 
mythologies vides, factices, non motivés est désigné du doigt. Les glissements du texte dans ce jeu ludique avec le sens qui se tapit dans la relation entre des signes, la "jouissance" [...] refusent la mimesis, empêchant donc toute identification simple, notamment autobiographique. » (Ramsay, 2010, p. 306). Ainsi, dans son film Glissements progressifs du plaisir, comme c'était déjà le cas dans la Justine du marquis de Sade, il y a de nombreux cadavres démembrés, mais cette exagération engendre une sorte de saturation : les bourreaux et les victimes se ressemblent, ils échangent leurs masques et le spectateur devient rapidement indifférent aux éventuels stimuli sexuels de la pellicule.

Cette interprétation est confirmée par le fait que, dans ses romans également, Robbe-Grillet n'a jamais cédé à un voyeurisme complaisant. Le regard, le principal canal perceptif dans son approche phénoménologique, ne parvient jamais à pénétrer le motif essentiel qui génère l'intrigue : un angle mort où (peut-être) un homicide s'est produit (Le Voyeur), où un mari jaloux peut trouver confirmation de l'infidélité de sa femme (La Jalousie). C'est la raison pour laquelle il est impossible de trouver des scènes pornographiques explicites dans ce qu'on appelle la «trilogie phénoménologique » (Les Gommes, 1953; Le Voyeur, 1955; La Jalousie, 1957). Dans ces romans, il y a toujours un obstacle qui bloque notre point de vue. Notre "vision entravée » (Brignoli) fait que nous sommes près de l' « intermittence » décrite par Barthes dans Le Plaisir du texte:

L'endroit le plus érotique d'un corps n'est-il pas là où le vêtement bâille? Dans la perversion (qui est le régime du plaisir textuel) il n'y a pas de "zones érogènes" (expression au reste assez casse-pieds); c'est l'intermittence, comme l'a bien dit la 
psychanalyse, qui est érotique : celle de la peau qui scintille entre deux pièces (le pantalon et le tricot), entre deux bords (la chemise entrouverte, le gant et la manche); c'est ce scintillement même qui séduit, ou encore: la mise en scène d'une apparition-disparition. (p. 17-18)

\section{Au-delà des limites du regard}

Dans la phase suivante de sa production, lorsque, au début des années 1960, comme l'affirme Renato Barilli, il passe de « Hussler à Roussel » (p. 12), Robbe-Grillet change radicalement son approche : de La Maison de rendez-vous (1965) à Souvenirs $d u$ triangle d'or (1978), il y a une prolifération de scènes de sexe et de torture. Cette révolution reflète la nouvelle tendance postmoderne. Dans ces textes, on trouve une prolifération de citations, mais il est très difficile de découvrir ce qui est original. Dans ce cas, le détachement ne passe pas par une approche phénoménologique, mais il y a plutôt un jeu intertextuel qui implique plusieurs genres: les histoires d'espionnage et, bien évidemment, la pornographie.

Pendant cette période, l'art expérimental paraît démystifier, à travers ces nouvelles mythologies, «l'inconscient collectif de la société » (Dumur, p. 47). En s'écartant de la phénoménologie et des suggestions de Hussler, Robbe-Grillet se tourne vers un paysage médiatique toujours plus vaste ${ }^{4}$.

Djinn (1981) représente son dernier effort dans cette direction, suivi de quatre ans de silence. Ensuite, Robbe-Grillet relève son plus grand défi: les Romanesques, une trilogie autobiographique. Il semble, enfin, vouloir dire la vérité

\footnotetext{
${ }^{4}$ Selon la formule de Ramsay (2010, p. 311), « le mythe, chez Robbe-Grillet, est [...] devenu la mythologie de Barthes ».
} 
concernant les liens qui existent entre ses fantasmes personnels et les fantasmes sadiques qui hantent ses romans et ses films. Avec un brin de malice, dans Le Miroir qui revient, il affirme : «Je n'ai jamais parlé d'autre chose que de moi » (p. 10). Suivant une pure ligne freudienne, il attribue la cause de ses fantasmes au traumatisme de son initiation sexuelle. Durant son enfance, le jeune Alain serait tombé amoureux d'une jeune fille un peu mystérieuse (ou vue comme telle), Angélique Arno. Mais celle-ci serait décédée tragiquement, en tombant d'un rocher... Voilà l'explication de l'écrivain. Mais il est difficile de lui faire confiance parce que, dans la trilogie des Romanesques, RobbeGrillet confond délibérément les trois instances (auteur; narrateur et personnage) de l'autobiographique idéale, théorisée par Philippe Lejeune. De surcroît, dans les Romanesques, il y a confusion entre le plan de la réalité, même déformée par le souvenir, et celui de la fiction. En effet, RobbeGrillet crée notamment, avec le personnage d'Henri de Corinthe, une sorte d'alter ego incarnant ses désirs les plus profonds et ses plus fortes craintes. Par conséquent, il est impossible de dire précisément qui parle de qui : comme dans le paradoxe du philosophe chinois Zhuangzi et du papillon, il est difficile de savoir, avec certitude, si Robbe-Grillet rêve de Corinthe, ou bien si c'est Corinthe qui imagine son créateur, dans une optique renversée qui ne manque pas de donner le vertige.

Une fois de plus, Robbe-Grillet offre au lecteur l'illusion de remonter à l'origine (origine biographique, origine psychologique, etc.) de ses obsessions, mais c'est pour détruire cette illusion. Selon lui, il n'y a pas de vérité définitive, pas de signification définitive en mesure d'arrêter le mouvement incessant de la signifiance : 
[...] il devient urgent de tout remettre en cause, et, replaçant les pions à leur point de départ, l'écriture à ses origines, l'auteur à son premier livre, de s'interroger à nouveau sur le rôle ambigu que jouent, dans le récit moderne, la représentation du monde et l'expression d'une personne, qui est à la fois un corps, une projection intentionnelle et un inconscient. (Robbe-Grillet, 1984, p. 12)

Encore une fois, l'invention linguistique l'emporte sur la présumée fidélité au référent ou, pour utiliser les mots de Seymour Chatman, le «discours» l'emporte sur l'« histoire». On ne peut pas trouver dans le monde extérieur (dans la réalité, dans nos corps...) l'élément impur que nous devons neutraliser parce qu'il est enraciné en nous-mêmes, dans notre langage :

Dans ces romans, le langage [...] est traité comme un corps, un corps de mot [...] dont la corporalité et la virtuosité sont sources de plaisir et de bonheur. La textualité et la sexualité forment un tout unique. (Stoltzfus, 1984, p. 192) ${ }^{5}$

Même dans le roman publié peu avant sa mort, Un roman sentimental (2007), Robbe-Grillet n'avait pas perdu son goût de la provocation. Dans ce "conte de fée pour adultes», nous est exposée l'éducation sexuelle de Gigi, une jeune fille de 14 ans perdue dans le Pays des merveilles du sexe et de la mort. Mais on est loin de l'innocence un peu niaise de la Justine sadienne. Gigi est probablement, malgré son âge, l'incarnation ultime du stéréotype de la femme fatale. Sachant que le contenu du livre peut troubler certains lecteurs, la maison d'édition, Fayard, a décidé d'ajouter un autocollant d'avertissement, en prévenant le lecteur qu'il s'agit d'une « fiction fantasmatique qui risque de heurter certaines sensibilités ». La critique, une fois de plus, fut

\footnotetext{
5 « Language in these novels [...] is treated like a body - a body of words [...] whose corporeality and resourcefulness are source of pleasure and delight. Textuality and sexuality are one. ». Nous traduisons.
} 
divisée (voir Milat, 2010). Beaucoup de commentateurs furent scandalisés par ce livre. En revanche, nous trouvons une opinion antithétique dans la recension parue dans The Guardian, intitulée Pornography as high art, qui débute avec la phrase suivante : «Le dernier roman d'Alain Robbe-Grillet est obscène et troublant, et d'autant plus à cause de son évidente capacité artistique » :

À partir de Sade, beaucoup d'écrivains français n'ont jamais cessé d'exploiter ce filon, isolé et inquiétant : Apollinaire, Louis Aragon, André Pieyre de Mandiargues... Robbe-Grillet, qui a maintenant 85 ans, n'est pas, comme certains critiques l'ont défini, simplement un autre vieux bonhomme dégoûtant, mais plutôt un innovateur dans ce filon dangereux et très français. Et ce qui peut être son dernier livre mérite d'être lu avec le plus grand soin. Provocation, titillation ou divertissement intellectuel? Je ne sais pas. Mais une chose est sûre : je ne vois aucun écrivain anglais ou américain qui oserait prendre un risque tellement impie. (Jakubowski) ${ }^{6}$

Encore une fois, c'est la limite entre érotisme et pornographie qui est remise en question. Comme nous l'avons déjà affirmé, cette limite varie puisqu'il s'agit du résultat d'une négociation sociale. Nous pouvons donc interpréter le travail de RobbeGrillet de la même manière que Barthes interprète le travail du marquis de Sade: "Son devoir, brillamment réalisé, vise à contaminer réciproquement l'érotique et la rhétorique, le discours et la criminalité » $(1980$, p. 33).

\footnotetext{
6 « Since Sade, many French writers have continued to mine this lonely and disturbing area: Apollinaire, Louis Aragon, André Pieyre de Mandiargues ... Robbe-Grillet, now 85, is not, as some critics have suggested, just another dirty old man, but another trailblazer on this perilous and very French road. And what could well be his final book should be read with the utmost care. Provocation, titillation or an intellectual divertissement? I remain uncertain. But one thing's for sure: I cannot imagine any English or American writer daring to take such an unholy risk. » Nous traduisons.
} 


\section{La catharsis et la mimesis}

Cette hypothèse nous conduit au cœur du problème - est-ce que la catharsis peut exister en dehors de la poétique de la mimésis? - et nous aide à évaluer l'efficacité de la défense de Robbe-Grillet. Ses arguments sont puissants, mais ils comportent quelques contradictions. Par exemple : comment peut-on concilier la réfutation de la tragédie - présentée comme un faux compromis entre l'homme et les choses exprimée dans Pour un nouveau roman avec la reprise, dans les Romanesques, du concept de la catharsis? Évidemment, la notion de catharsis peut légitimer certains contenus outrageants, même à notre époque moderne, mais nous devons considérer cette hypothèse au sein d'un paradigme idéologique spécifique. Dans la Poétique d'Aristote, la catharsis renforce la cohésion sociale, en purgeant la société de ses passions dangereuses. Dans la vision de Robbe-Grillet, l'art a une mission différente: démystifier l'idéologie bourgeoise. Pendant les années 1960, en particulier, cette déconstruction des nouvelles mythologies acquiert un caractère critique et politique :

Robbe-Grillet joue avec les images de mythes culturels : il les expose, il les rend visibles, il les dissèque, tout comme il démembre les corps des filles et des mannequins qui peuplent ses romans et ses films; l'érotisme et la violence de ses œuvres, à côté de ses nuances culturelles, représentent une attaque contre la langue et contre la doxa; jouer avec le corps du texte ou avec les corps dans le texte signifie affirmer une dimension de liberté qui était niée par le langage, la "nature" et la société. (Stoltzfus, p. 199) ${ }^{7}$

\footnotetext{
7 «Robbe-Grillet toys with the images of cultural myths: exposing them, rendering them visible, dissecting them, even as he dismembers the bodies of the girls and manikins that inhabit his novel and films, The eroticism and the violence of his works, in addition to the cultural overtones, are and assault on
} 
Plusieurs critiques ont contesté cette position, déclarant que l'exhibition des fantasmes sadomasochistes qui hantent l'inconscient collectif, même à travers la parodie et l'intertextualité, ne neutralise pas leur fascination perverse. Ainsi, Raylene Ramsay s'interroge :

Est-ce que les images de l'autosuffisante sexualité féminine comme consentante au voyeurisme, à sa propre défloration ou mise à mort, ou jouant sur sa relation « incestueuses » de petite fille avec le père puissant [...] sont chez Robbe-Grillet complètement ludiques, mises à plat? [...] Son œuvre est-elle vraiment le jeu froid, "the cool detached surfaces» d'une certaine forme de l'intertextualité, du postmoderne? » (2010, p. 307)

La question peut être reformulée de la manière suivante: comment l'utilisation de stéréotypes véhiculés par les médias peut-elle détruire l'idéologie qui les a générés? Ramsay poursuit :

Nature, humanisme, crime, condamnations et fantasmes sexuels personnels peuvent être [...] exposés en tant que représentations rendues inoffensives mais [...] dans le processus d'exposition, elles n'ont pas perdu tout leur pouvoir. Leurs significations ont été rayées, mais pas effacées, elles ont été mises " en réserve » mais pas détruites. ${ }^{8}$ (1992, p. 142)

En dépit des théories du village planétaire mises en avant par Marshall McLuhan, il est difficile de croire que nous pouvons changer notre conception du monde simplement en jouant avec

langue and doxology; to play with the body of the text or the bodies in the text is to assert a dimension of freedom that language, "nature" and society otherwise deny. » Nous traduisons.

${ }^{8}$ « Nature, humanism, crime and punishment, and personal sexual phantasies may be [...] ex-posed as harmless representations but [...] in the process of exposure, they have not lost all power. Their meanings are struck out but not erased, the have been placed "in reserve" (or could we say "under erasure"?) but not destroyed. Nous traduisons. 
nos anciennes conventions artistiques. Nous ne pouvons pas accepter la notion traditionnelle de catharsis parce que l'art joue un rôle différent dans notre société : l'art ne renforce pas la cohésion sociale, mais vise à renverser le statu quo. Donc, il n'existe pas de réconciliation possible entre ces deux perspectives ou, en d'autres termes, entre esthétique et morale.

Ce n'est qu'en nous penchant sur l'aspect psychologique de la question que nous pouvons comprendre la position de RobbeGrillet, notamment quand il affirme :

[...] je me serais mis à écrire des romans pour exorciser ces fantômes dont je ne venais pas à bout, et [...] le biais de la fiction est, en fin de compte, beaucoup plus personnel que la prétendue sincérité de l'aveu. (1984, p. 16-17)

Cette confession inhabituelle explique la raison pour laquelle la transmutation des fantasmes en objets esthétiques et/ou conceptuels est réalisée à travers un jeu intertextuel, mais la personne de l'auteur ne peut pas rester en marge de ce processus.

L'enjeu est vraiment de taille : " il s'agit d'exploiter (au lieu de les camoufler) les intermittences de la mémoire et les mécanismes du refoulement qui engloutissent le vécu danse les abîmes du néant, à moins de le faire émerger par un effort d'élaboration, au moyen de l'association et/ou de l'écriture » (Devésa, p. 295). Dans cette perspective, l'art pourrait exorciser nos démons intérieurs. Chacun, en affrontant ses démons, ses fantasmes, ses obsessions, est placé devant deux choix - et, là, on peut sans doute trouver une connexion entre l'individu et la société - : la répression ou l'exhibition. Robbe-Grillet préfère la seconde option et, selon lui, ses accusateurs puritains devraient faire le même choix : 
S'ils avaient, au contraire dès l'adolescence, grâce à un autre type d'éducation, fréquenté leur propre face cachée, c'est-à-dire ces crimes latents qu'ils portaient en eux, ils auraient vite appris à les reconnaître, pour les examiner à loisir, puis à les dominer, et bientôt à en jouir sans honte, sans risquer non plus d'attenter un soir à la vie d'autrui ni à sa liberté. (1987, p. 197)

De son point de vue, la catharsis ne renforce pas la cohésion sociale en proposant et en confirmant un ensemble stable de valeurs partagées. L'artiste et son public ne peuvent que jouer avec leurs fantasmes, mais sans jamais les effacer ou les purifier. En refusant toute forme de censure ou de contrôle, Robbe-Grillet, reprenant les mots de Breton) conclut: «la pornographie est l'érotisme des autres » (Évrard, p. 12).

Cette affirmation évoque un aspect particulier du problème, mais ne propose pas un équilibre différent et satisfaisant parmi les trois perspectives prises en compte au début de notre étude. $\mathrm{Si}$, comme l'affirme Foucault, la sexualité, à l'époque moderne, n'est pas simplement réprimée, mais enfermée dans des formes spécifiques du discours, nous devons comprendre pourquoi et comment ces différents discours interagissent les uns avec les autres.

L'artiste moderne, constamment poussé, à travers ses travaux, vers l'horizon des aspirations, ne peut pas, sans hésitation, accepter la distinction entre érotisme et pornographie comme une affirmation de facto. D'autre part, les représentants des institutions donnent l'impression de considérer la pornographie de manière plutôt différente. Pendant de nombreuses décennies, l'accusation d'obscénité a été déterminée par le contenu (ce qui est représenté est troublant) et par les effets sociaux, présumés, sur le public (la pornographie «déprave et corrompt ceux dont l'esprit est 
ouvert à ce genre d'influences immorales », Ducat, p. 982). Ce n'est que récemment que l'obscénité a été définie dans un contexte précis, en relation avec les «normes de la communauté ». Le "test de Miller», introduit aux États-Unis dans les années 1970, représente un bon exemple de cette approche innovante: une œuvre n'est considérée comme obscène que si l'on y trouve ces trois conditions :

(a) si la personne moyenne, en appliquant certaines normes contemporaines de la communauté, trouve que l'œuvre, prise dans son ensemble, fait appel à l'intérêt le plus lascif;

(b) si l'œuvre dépeint/décrit, de manière clairement offensante, des conduites sexuelles spécifiquement définies par la loi applicable de l'État;

(c) si l'œuvre, prise dans son ensemble, manque de sérieuses valeurs littéraires, artistiques, politiques ou scientifiques. ${ }^{9}$ (U.S. Supreme Court)

Comparés à la mentalité apparemment étriquée du tribunal de Venise, les juges américains paraissent plus ouverts (en considérant, en particulier dans le deuxième point, la relation entre le contenu et la forme), mais même la solution qu'ils proposent présente des faiblesses évidentes.

Dans de nombreux cas, on peut distinguer l'érotisme et la pornographie en prenant en considération l'opinion de la "personne moyenne» mais, quand on pense à l'art expérimental, cette même opinion peut être trompeuse. L'art

9 «(a) whether the average person, applying contemporary community standards, would find that the work, taken as a whole, appeals to the prurient interest; (b) whether the work depicts/describes, in a patently offensive way, sexual conduct specifically defined by applicable state law; (c) whether the work, taken as a whole, lacks serious literary, artistic, political or scientific value. » Nous traduisons. 
expérimental n'entre pas dans l'horizon commun des aspirations et tend, par sa nature propre, à aller au-delà de cette limite. Donc, dans ce cas, comment la valeur artistique peut-elle être déterminée par le sens commun?

Le problème est double. Tout d'abord, le test de Miller implique la présence d'entités et de standards hypothétiques ( "la personne moyenne», le « sens commun », etc.) en tant que derniers arbitres, mais ces entités abstraites ne peuvent pas être définies de manière prescriptive. Elles impliquent une forme particulière et une certaine consistance quand elles sont en discussion, quand il y a un scandale ou un conflit entre différents codes. Donc, si nous supposons que ces entités et standards existent, ils peuvent représenter une référence valable pour notre jugement?

Selon Robbe-Grillet, il n'en est pas question. Sa définition de la pornographie est relative et subjective ou, en d'autres termes, elle est une définition par négation. Il ne s'agit pas d'une définition basée sur la doxa ("sens commun»), mais contre la doxa. En suivant le chemin tracé par Foucault, Robbe-Grillet arrive à briser « le régime de pouvoir-savoir-plaisir qui soutient chez nous le discours sur la sexualité humaine » (Foucault, p. 19) et les accusations d'obscénité que nous avons relevées contre son œuvre ne sont pas honteuses, mais elles représentent la preuve de son succès. 


\section{Bibliographie}

BARILLI, Renato. (1998), Robbe-Grillet e il romanzo postmoderno, Milan, Mursia.

BARTHES, Roland. (1980), Sade, Fourier, Loyola, Paris, Seuil.

—. (1973), Le Plaisir du texte, Paris, Seuil.

BrignOLI, Laura. (2002), «La technique "privative" du Nouveau Roman: l'exemple de Robbe-Grillet», Francofonia, $n^{\circ} 43$, automne, p. 57-76.

Chatman, Seymour. (1978), Story and discourse: narrative structure in fiction and film, Ithaque-Londres, Cornell University Press.

DEVÉSA, Jean-Michel. (2010), « CEdipe au miroir de Thanatos », dans Roger-Michel AllEMAND et Christian Milat (dir..), Alain Robbe-Grillet. Balises pour le XXIe siècle, Paris / Ottawa, Presses Sorbonne Nouvelle / Presses de l'Université d'Ottawa, p. 292-303.

DuCAT, R. Craig. (1996), Constitutional interpretation, Minneapolis/St. Paul, West Pub Co.

Dumur, Guy. (1970), «Le sadisme contre la peur», Le Nouvel Observateur, no 310, 19-25 octobre, p. 47-49.

Eco, Umberto. (1994), Il secondo diario minimo, Milan, Bompiani.

ÉVRARD, Franck. (2003), La Littérature érotique ou l'écriture du plaisir, Paris, Éditions Milan.

FouCAulT, Michel. (1976), La Volonté de savoir, Paris, Gallimard. 
HEnRIOT, Émile. (1955), " Le Prix des Critiques: Le Voyeur, d'Alain Robbe-Grillet », Le Monde, 15 juin, p. 9.

JAKUBOWSKI, Maxim. (2007), «Pornography as high art», Guardian.co.uk. Books blog, 18 décembre, http://www.guardian.co.uk/books/booksblog/2007/dec/1 8/pornographyashighart

MAZzocuT-Mis, Maddalena. (2008), Estetica della fruizione, Milan, Lupetti.

Milat, Christian. (2010), «Un roman sentimental: le dernier "nouveau roman" robbe-grillétien?», dans Roger-Michel AllEMAND et C. Milat (dir..), Alain Robbe-Grillet. Balises pour le XXIe siècle, Paris / Ottawa, Presses Sorbonne Nouvelle / Presses de l'Université d'Ottawa, p. 483-491.

NEWTON, Elisabeth. (2005), "Perception and Power: the Phenomenology of Sexuality in Robbe-Grillet's Film and Fiction », dans S. DONANCHIE et K. HARRISON (dir.), Love and Sexuality: New Approaches in French Studies, Oxford, Peter Lang, p. 121-142.

RobBe-Grillet, Alain. (1987), Angélique ou l'enchantement, Paris, Minuit.

—. (1984), Le Miroir qui revient, Paris, Minuit.

-. (1963), Pour un nouveau roman, Paris, Minuit.

RAMSAY, Raylene. (2010), « Le nouvel CEdipe : jeux et enjeux de la violence sexuelle chez Robbe-Grillet »; dans Roger-Michel ALLEMAND et Christian Milat (dir..), Alain Robbe-Grillet. Balises pour le XXIe siècle, Paris / Ottawa, Presses Sorbonne Nouvelle / Presses de l'Université d'Ottawa, p. 303-315. 
-. (1992), Robbe-Grillet and Modernity. Sex, Sexuality and Subversion, Gainesville, University of Florida Press.

SARRAUTE, Nathalie. (1956), "Ce que voient les oiseaux», dans L'Ère du soupçon, Paris, Gallimard, coll. « Folio Essais ».

Stoltzfus, Ben. (1984), «The Body of Robbe-Grillet's Text: Myth, Sex and Politics in the Nouveau Nouveau Roman " Neophilologus, $\mathrm{n}^{\circ}$ 2, vol. 68, p. 192-205.

U.S. Supreme Court. (1973), Miller v. California, $<$ http://caselaw.lp.findlaw.com/cgibin/getcase. pl? court $=$ us\&vol=413\&invol=15>.

\section{Résumé}

Une ligne subtile sépare l'érotisme de la pornographie dans la production artistique d'Alain Robbe-Grillet. La réception de son œuvre nous indique que la relation entre ces deux notions est le résultat d'une négociation sociale impliquant différents codes (code esthétique, code légal, etc.). Nous appuyant sur certains épisodes de la carrière de Robbe-Grillet (l'accusation d'obscénité portée contre son film, Glissements progressif $d u$ plaisir, autour des années 1970; la controverse engendrée par la publication de son dernier roman, Un roman sentimental, 2007), nous voulons montrer pourquoi et comment cette négociation peut amener à des sérieuses incompréhensions et, à la limite, échouer. 


\begin{abstract}
A thin line separates eroticism and pornography in Alain Robbe-Grillet's artistic production. Examing the reception history of his work we can parse out the relation between those two notions as the result of a social negotiation that involves different codes (aesthetic code, legal code, etc.). Focusing our attention on a few episodes in Robbe-Grillet's career (the obscenity case against the movie Glissements progressif $d u$ plaisir in the Seventies; the controversy aroused by the last novel published before his death: Un roman sentimental, 2007) we want to show why and how this negotiation could lead to serious misunderstandings and, at worst, fail.
\end{abstract}

